\title{
Evaluation of punctuality on a heavily utilised railway line with mixed traffic
}

\author{
O. Lindfeldt \\ Division of Traffic and Logistics, Department of Transport and \\ Economics, Royal Institute of Technology, Stockholm, Sweden
}

\begin{abstract}
The Western Main Line (450 km) between Stockholm and Gothenburg is heavily utilised for mixed rail traffic. High speed trains are mixed with regional, local and freight trains and the congestion clearly affects the overall punctuality. In order to evaluate the possible total effects of reduced primary delays, the high speed operator, $\mathrm{SJ} \mathrm{AB}$, took the initiative to perform an extensive simulation study in RailSys, which was carried out by the Royal Institute of Technology.

As preparation for this simulation study delay data was compiled for all trains using the line. This paper briefly discusses the ideas of stochastic disturbances in the simulation of rail operation and also the compilation methods for primary delay distributions.

The resulting distributions from the Western Main Line were then analysed. It was clearly shown that the primary delays are extensive and have a high degree of variability. The paper ends with a discussion about the validation of the simulation model and possibilities to develop methods for more accurate stochastic modelling.
\end{abstract}

Keywords: simulation, delay distribution, perturbations, mixed traffic.

\section{Introduction}

The demand for rail passenger traffic and freight transport is steadily increasing in Sweden and the utilisation has now become troublesome in many sections of the railway network. The Western Main line $(450 \mathrm{~km})$, connecting Stockholm and Gothenburg, is one of the most heavily utilised lines. On this line the traffic consists of an unfavourable mix of high speed $(200 \mathrm{~km} / \mathrm{h})$, regional and local 
passenger trains and freight trains. There are many level crossings and numerous junctions with connecting trains resulting in strong dependencies between trains.

Primary delays are frequent and due to the congestion these are often propagated to other trains in the proximity. Several sections of the line have such high utilisation that overall punctuality is affected negatively. The high speed trains are particularly sensitive to all kinds of disturbances and this is clearly reflected in the delay statistics.

The operator of the high speed trains, SJ AB, has paid special attention to the punctuality problems. In order to evaluate the overall effects of reduced primary delays caused by the operator itself, including vehicle failures, an extensive simulation project has been performed by the Royal Institute of Technology.

The simulation, performed in RailSys, included all trains on the Western Main Line. A reference case was defined as the autumn 2006 timetable period with 105 weekdays with the same timetable. Delay data from this period was used to produce delay statistics for the simulation. This extensive preparation work, including initial delays, as well as delays on every line section and dwell time extensions, generated important results in itself.

In this paper the simulation method is described briefly as a background to the delay evaluation that is then treated in more detail. A simple method for isolating primary delays from total delays is discussed and statistics for initial delays, dwell time extensions and run time extensions are presented.

The paper ends with some conclusions and ideas for future work on heavily utilised railway lines with mixed traffic.

\section{Method and modelling}

The idea of the project was to evaluate the effect of reduced primary delays, first and foremost for the high speed trains. To do this, the infrastructure of the line was built up in RailSys and the reference timetable, with approximately 900 trains, was applied.

\subsection{Model}

The delays in rail operation may be subdivided into groups according to the source. In Sweden delays are often divided into:

- Infrastructure related delays

- Operator related delays

- Vehicle related delays

- Other, not specified delays

- Secondary delays

These groups are used in the report system (TFÖR), where the causes of delays longer than four minutes are reported manually by the dispatchers. These reports were used to subdivide the delays (see the next section).

In this project the first three groups were of special interest. Infrastructure failures cause primary delays to all types of trains, whereas operator and vehicle related delays primarily affect the trains of the operator concerned. 


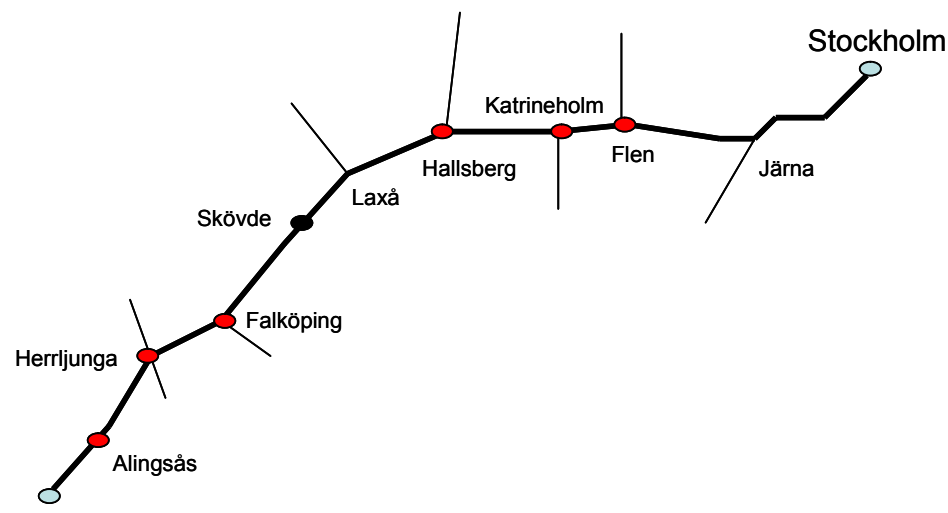

Gothenburg

Figure 1: Western main line.

All primary delays were modelled as independent perturbations in the simulation. However, this is a simplification compared to real situations and careful validation is therefore required, during which steering parameters such as dispatching rules etc are adjusted.

In the simulation all kinds of deviations from the timetable were handled through disturbances generated by delay distributions. This means, for example, that all infrastructure sections (points, signals etc) were assumed to be available all the time. The effect of infrastructure failures was instead caught by run time extensions given by applied distributions. This type of indirect modelling naturally assumes that the applied distributions contain only primary delays.

\subsection{Isolation of primary delays}

In the train operation database (TFÖR) all trains are registered at each station. The registered time is compared to the scheduled time and the deviation is stored in the database, making it possible to compile delay distributions and other statistics from historical data.

This was done for all trains during the timetable period (autumn 2006). The passenger trains were treated in groups according to patterns given by basic interval timetables, whereas the freight trains were analysed individually in the first step and then grouped according to mean and variance.

In order to distinguish between different types of delay causes, the reports of causes were used to separate different delay types. This work resulted in primary delay distributions for each group of trains. The method was also used to exclude selected parts of delays caused by infrastructure, operator and vehicle respectively. This was a preparation for the simulations that evaluated the effect of specific reductions of primary delays. 
This type of reduction of delay data faced two major problems:

- Accuracy of the manual delay reports

- Lack of reports for delays shorter than five minutes.

No other sources of information about the delay causes were available and the reports were therefore accepted without further investigation. The lack of information about delays shorter than five minutes was handled in a special way. The secondary delays between three and five minutes were assumed to have the same proportion to the total delays as in the 5-9 minutes' delay interval. All delays of 1-2 minutes were assumed to be primary.

This way of estimating delays is probably a rough assumption that should be examined carefully before it is more widely used, but was assumed to be an acceptable first approximation.

\section{Disturbances}

The stochastic part of the rail operation was modelled in RailSys by three different types of disturbances:

- Initial delays

- Run time extensions

- Dwell time extensions

The first two were derived from historical delay statistics as described above, whereas dwell time extensions were estimated by measurements on platforms.

\subsection{Initial delays}

Initial delays were compiled for all trains at their first station on the studied line. Trains belonging to the same basic interval timetable-concept were treated as a group. Fig. 2, 3 and 4 show the result when each distribution is represented by its mean and standard deviation values.

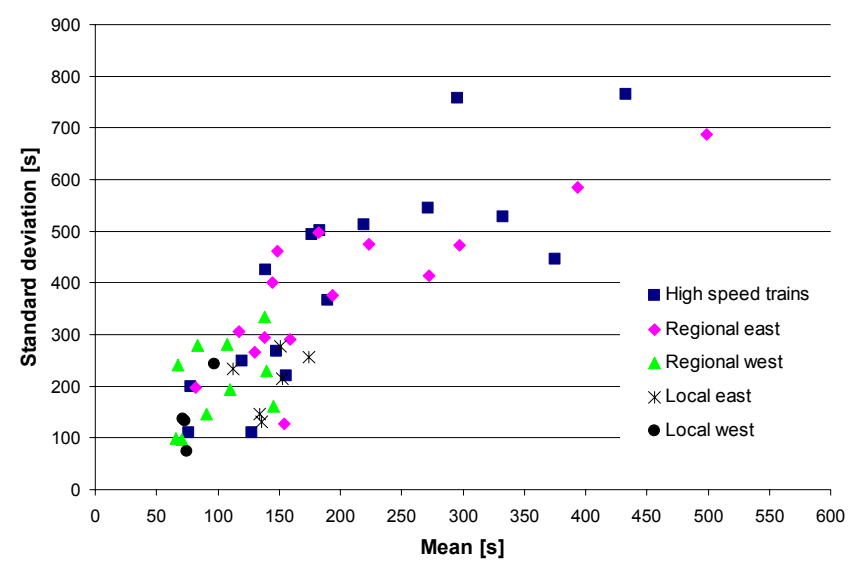

Figure 2: Characteristics of passenger trains' initial delays. 


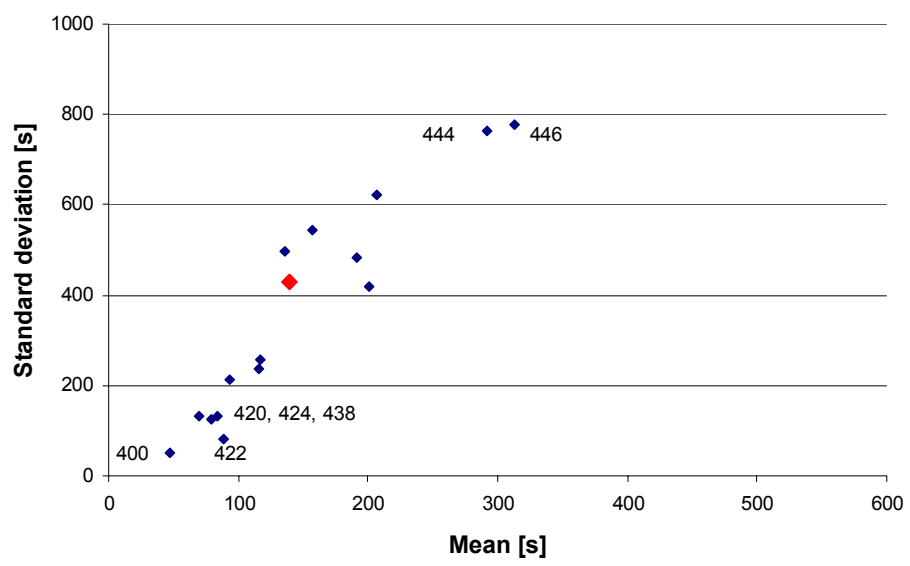

Figure 3: Characteristics of individual high speed trains. Each point represents one train. Some trains are labelled with their train number.

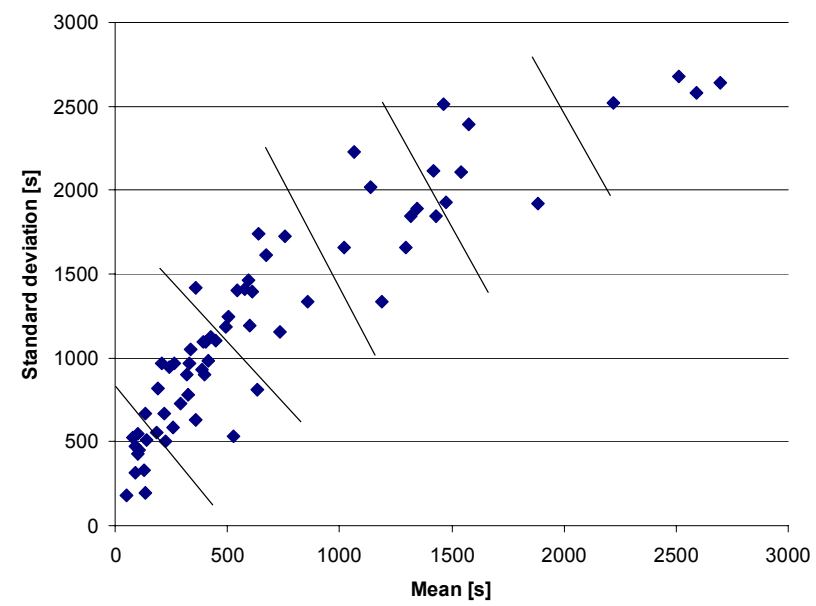

Figure 4: Characteristics of freight trains' initial delays.

Fig. 2 shows that the high speed trains have the worst punctuality of the passenger trains. Both mean delay and standard deviation are high for the most important high speed train groups. A few high speed train patterns have better distributions, located closer to the origin. Unfortunately, most of these groups consist of single peak hour trains travelling to or originating from smaller cities near to the Western Main Line.

Regional trains running on the eastern part of the line show high delays. This is partly explained by systematic vehicle problems during the timetable period, 
but also by congestion effects in the Stockholm area. Regional trains running on the western part of the line and local trains show less delay.

In order to show that the trains within one and the same group behave differently the distribution for each individual train (105 observations) was compiled. Fig. 3 shows an example of this: up-going high speed trains from Gothenburg to Stockholm. Typically, trains running early (numbers 400 and 422-424) have less delay than trains running late (numbers 444 and 446). Possible explanations for this are accumulation of delays due to low time supplements and short turnaround times compared to the overall delay level combined with a higher capacity utilisation in the afternoon. In the simulation the whole group was represented by a distribution indicated by the larger diamond in the middle.

The freight trains were evaluated individually, see fig. 4, and then grouped into six groups according to mean and standard deviation. In this compilation trains that arrived early were treated as on-time trains. As can be seen from the figure, some trains have clear punctuality problems. Only the first group, consisting of mail trains etc, show the same levels as the passenger trains.

\subsection{Run times}

All kinds of primary delays occurring between ordinary passenger stops (origin and destination station for freight trains) were modelled as run time extensions. To apply this idea, the line was divided into 13 sections, each approximately 35 $\mathrm{km}$ long. For each section, direction and train type, a distribution for the extension was compiled from delay data.

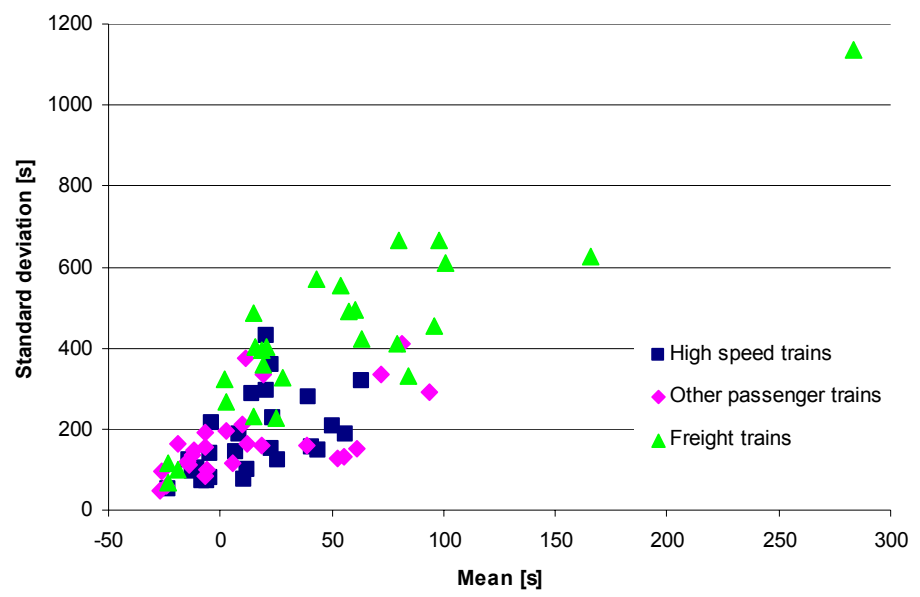

Figure 5: Characteristics of run time extensions: 3 train types, 2 directions and 13 sections.

This was done through comparison of the arrival delay at the end station of each section and the departure delay at the first station. Delay cause reports were used to exclude secondary delays in the same way as described previously. 
In some cases the mean extension was found to be negative, which means that the trains on these sections, on average, run faster than their timetabled run time.

\subsection{Dwell times}

Different types of primary delays also occur in connection with ordinary passenger stops. To examine and model these effects SJ AB performed manual measurements of passenger stops for high speed trains. This empirical data was then fitted to lognormal distributions with quite good agreement.

The dwell time may be subdivided into two parts: exchange time, i.e. the time that is needed for door opening, alignment and boarding, and technical time for door closing. Fig. 6 shows the resulting distributions for the exchange time. To this exchange time a shortest technical time must then be added, which is vehicle dependent and was measured to be 30 seconds for the high speed vehicle X2. The bold vertical line represents the remaining timetabled exchange time when the technical time is subtracted from the timetabled dwell time of two minutes.

It is clearly seen that the stops at Skövde (Sk), having the highest mean and standard deviation values, often exceed the timetabled dwell time (30-35\% of the cases), whereas the timetabled time is much better adjusted in Södertälje (Söö). At stations like Skövde, with a high variance in exchange time, it becomes quite difficult to choose a feasible timetable dwell time that works.

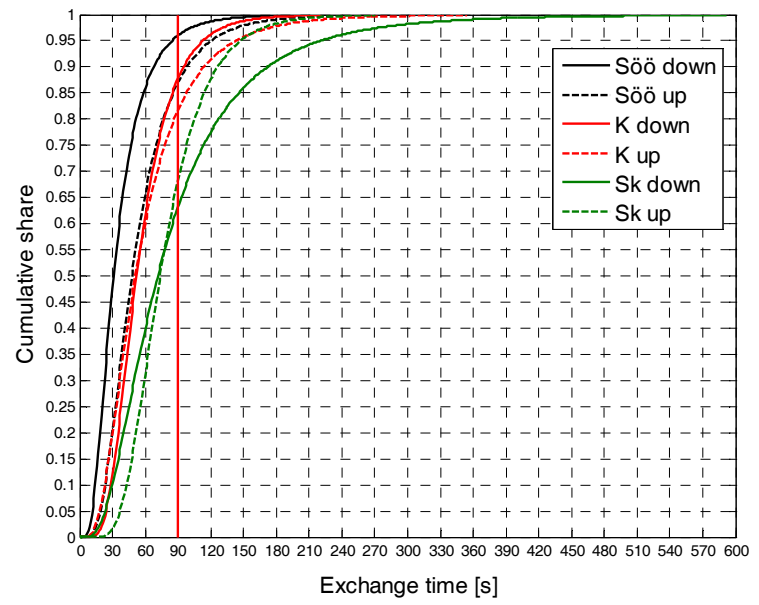

Figure 6: Distributions for passenger exchange time at three different stations.

\section{Results and validation}

Altogether, the compilation work resulted in 40 different empirical delay distributions that were used to validate the reference case. In the iterative 
validation process, simulated results were compared to real delay statistics for several stations along the line.

The validation focused on the high speed trains. Adjustments were made regarding:

- Dispatching priorities between different train types

- Trains' possibilities to make up delays by using timetabled supplements

- The highest values of run time extensions.

In Sweden a kind of "On-time-rule" is used when conflicts occur between different trains. This dispatching rule implies that a train that follows its timetable is to be prioritised over a delayed (conflicting) one. The high speed trains therefore also risk additional (secondary) delays once they are delayed.

A general experience from simulations in RailSys is that trains often make up delays using timetable supplements. The idea of run time extensions is to balance this effect. Despite a large number of run time extensions the possibilities to make up delays had to be limited on some line sections to obtain an acceptable agreement.

The highest values of run time extensions ( $>30$ minutes) turned out to be unrealistically high, causing more secondary delays in the simulation than in reality. Values over 30 minutes were therefore excluded from the run time extension distributions.

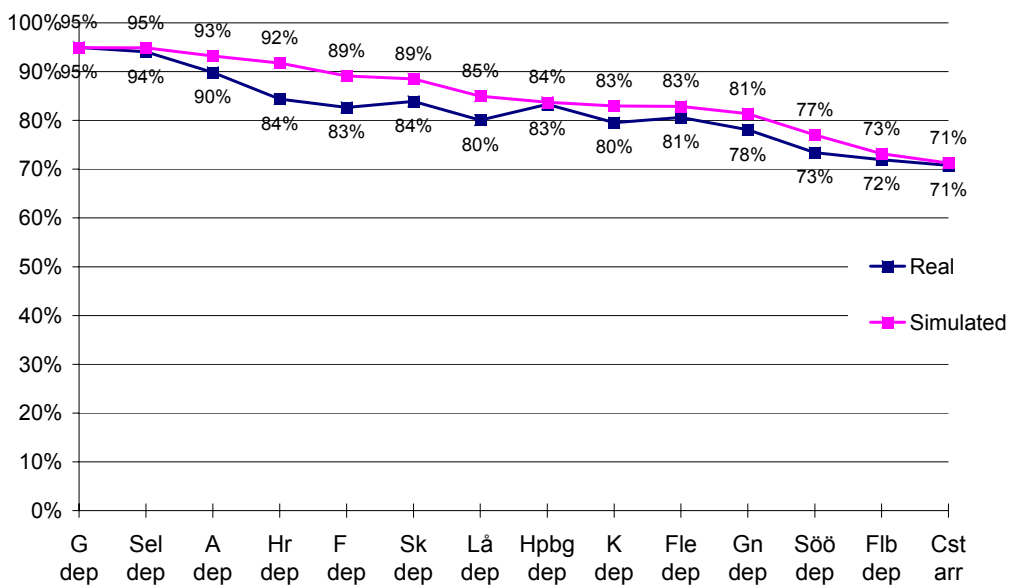

Figure 7: Example of validation results. Departure punctuality $(\leq 5 \mathrm{~min})$ for real and simulated up-going trains from Gothenburg (G) to Stockholm (Cst).

Fig. 7 shows an example of validation results. Despite calibration efforts the simulated result is significantly better than the real one. A sample of trains from other train groups was also tested in order to check that also these were also modelled properly. 


\section{Conclusions and further work}

This work showed that it is possible to model most of the stochastic behaviour of rail operation using three types of disturbances: initial delays, run time extensions and dwell time extensions. This method means a gathered approach where all kinds of stochastic behaviour are represented by a few distributions.

In order to examine the contribution of specific sources of disturbances it is therefore necessary to find out how different sources contribute to these collected distributions. This is a natural step for further evaluation. Such a separation was also performed within this project and the validated model was then used to examine effects of reduced primary delays.

The main results from this examination was that the high speed trains constitute such a limited share of the total number of trains, that reduced primary delays for just these trains does not affect overall delays very much.

The work also resulted in a deeper understanding of the delay situation on one of the most important lines in Sweden as regards both train groups and individual trains.

A simplified validation method using only one point of the delay cumulative distribution function ( 5 minute delay level) was applied in this project. A natural way to make the validation more accurate is to compare several points of the distributions and/or to compare means and standard deviations. The validation should also involve more than one train type. A check of the slower freight trains would reveal how the model treats trains with different features in dispatching situations.

\section{Acknowledgements}

This paper is an attempt to present some ideas for the important preparatory work with disturbance data which precedes all rail operation simulations. The simulation commissioned by SJ AB provided a good opportunity to learn more about delay statistics and compilation of disturbance data.

\section{Reference}

[1] Nelldal, B-L.et.al., Förbättrad punktlighet på X2000 - analys med hjälp av simulering, KTH 2007 